Bernhard Blanke - Stephan von Bandemer - Frank Nullmeier Göttrik Wewer (Hrsg.)

Handbuch zur Verwaltungsreform 
Bernhard Blanke

Stephan von Bandemer

Frank Nullmeier

Göttrik Wewer (Hrsg.)

\section{Handbuch zur \\ Verwaltungsreform}

3., völlig überarbeitete und erweiterte Auflage 


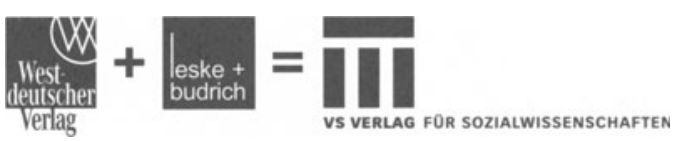

VS Verlag für Sozialwissenschaften

Entstanden mit Beginn des Jahres 2004 aus den beiden Häusern

Leske+Budrich und Westdeutscher Verlag.

Die breite Basis für sozialwissenschaftliches Publizieren

Bibliografische Information Der Deutschen Bibliothek

Die Deutsche Bibliothek verzeichnet diese Publikation in der Deutschen Nationalbibliografie;

detaillierte bibliografische Daten sind im Internet über <http://dnb.ddb.de> abrufbar.

2. Auflage 2001

3. Auflage Januar 2005

Alle Rechte vorbehalten

(c) VS Verlag für Sozialwissenschaften/GWV Fachverlage GmbH, Wiesbaden 2005

Der VS Verlag für Sozialwissenschaften ist ein Unternehmen von Springer Science+Business Media. www.vs-verlag.de

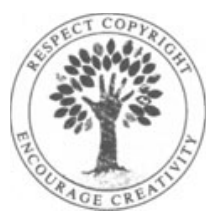

Das Werk einschließlich aller seiner Teile ist urheberrechtlich geschützt. Jede Verwertung außerhalb der engen Grenzen des Urheberrechtsgesetzes ist ohne Zustimmung des Verlags unzulässig und strafbar. Das gilt insbesondere für Vervielfältigungen, übersetzungen, Mikroverfilmungen und die Einspeicherung und Verarbeitung in elektronischen Systemen.

Die Wiedergabe von Gebrauchsnamen, Handelsnamen, Warenbezeichnungen usw. in diesem Werk berechtigt auch ohne besondere Kennzeichnung nicht zu der Annahme, dass solche Namen im Sinne der Warenzeichen- und Markenschutz-Gesetzgebung als frei zu betrachten wären und daher von jedermann benutzt werden dürften.

Umschlaggestaltung: KünkelLopka Medienentwicklung, Heidelberg

Gedruckt auf säurefreiem und chlorfrei gebleichtem Papier

ISBN 978-3-8100-4082-4

DOI 10.1007/978-3-531-90340-8

ISBN 978-3-531-90340-8 (eBook) 


\section{Inhalt}

Bernhard Blanke, Stephan von Bandemer, Frank Nullmeier und Göttrik Wewer

Vorwort

Bernhard Blanke

Verwaltungsreform als Aufgabe des Regierens - Einleitung

\section{Staats- und Verwaltungsverständnis}

Franz-Xaver Kaufmann

Zur historischen und aktuellen Entwicklung des europäischen Staates

Hubert Heinelt

Vom Verwaltungsstaat zum Verhandlungsstaat

Arthur Benz

Verwaltung als Mehrebenensystem

Stephan von Bandemer und Josef Hilbert

Vom expandierenden zum aktivierenden Staat

Gunnar Folke Schuppert

Zur Rolle des Rechts bei der Staats- und Verwaltungsreform

Carl Böhret

Verwaltungspolitik als Führungsauftrag

Werner Jann

Verwaltungswissenschaft und Managementlehre 


\section{Reform- und Managementkonzepte}

Eckhard Schröter und Hellmut Wollmann

New Public Management

Werner Jann

Neues Steuerungsmodell

Manfred Röber

Aufgabenkritik im Gewährleistungsstaat

Isabella Proeller und Kuno Schedler

Verwaltung im Gewährleistungsstaat

Hermann R. Bolz

Rechtsvereinfachung und Entbürokratisierung

Frank Nullmeier

Wettbewerb und Konkurrenz

Christoph Strünck und Rolf G. Heinze

Public Private Partnership

Jörg Bogumil und Lars Holtkamp

Bürgerkommune

Pia Karger, Oliver Rüß und Nadia vom Scheidt

E-Government

\section{Organisationsentwicklung}

Dominik Böllhoff und Göttrik Wewer

Zieldefinition in der Verwaltung

Werner Jann

Hierarchieabbau und Dezentralisierung

Tanja Klenk und Frank Nullmeier

Leitungsstruktur und Reformen der Leitungsorganisation

Matthias Dreyer und Walter Richter

Dezentrale Organisationsformen im Neuen Steuerungsmodell

Stephan von Bandemer und Michael R. Hübner

Prozessmanagement in der öffentlichen Verwaltung 
Bernd Andersch und Volker Belzer

Projektmanagement

Lothar Beyer

Informations- und Kommunikationstechnik

Matthias Dreyer und Walter Richter

Wissensmanagement

Henning Schridde

Verwaltungskultur, Change Management und lernende Organisation

\section{Personalentwicklung}

Christoph Reichard

Personalmanagement

Reinhard Rieger

Dienstrechtsreform

Nathalie Behnke

Alte und neue Werte im öffentlichen Dienst

Hans-Gerd Ridder und Frank Schirmer

Führung

Siegfried Mauch

Neue Wege der Personalrekrutierung

Hans-Gerd Ridder

Materielle und immaterielle Leistungsanreize

Sybille Stöbe-Blossey

Mitarbeiterbeteiligung

Jörg Semmler und Göttrik Wewer

Mitarbeitergespräche

Hermann Palm

Verwaltungsreform und Mitbestimmung

Hans-Jürgen Bruns und Hans-Gerd Ridder

Qualifizierung und Fortbildung 
Kerstin Ohlde und Marion Olthoff

Verwaltungsmodernisierung und Gender Mainstreaming

Hermann Palm

Kosten des öffentlichen Dienstes

\section{Budgetentwicklung}

Hansjürgen Bals

Neue Haushaltssteuerung

Heinrich Mäding

Öffentliche Haushalte zwischen demokratischer Steuerung und administrativer Effizienz

Lothar Beyer und Hans Georg Kinzel

Öffentliches Rechnungswesen: Kameralistik oder Doppik?

Bernd Adamaschek

Kosten- und Leistungsrechnung für den öffentlichen Sektor

Volker Belzer

Konsolidierung der Haushalte

Ulrich Schneider und Olaf Hugenberg

Neue Finanzierungsformen für öffentliche Aufgaben

Hermann Butzer

Wirtschaftlichkeit im Verwaltungsrecht

Wolfgang Göke und Heinz Thörmer

Öffentliche Finanzkontrolle durch Rechnungshöfe

\section{Ergebnis- und wirkungsorientierte Steuerung}

Kuno Schedler

Verwaltungscontrolling

Karsten Balzer

Produkte als Informationsträger

Frank Nullmeier

Output-Steuerung und Performance Measurement 
Stephan von Bandemer

Benchmarking

Stephan von Bandemer

Qualitätsmanagement

Götz Konzendorf

Gesetzesfolgenabschätzung

\section{Erfahrungen, Bewertungen und Perspektiven der Verwaltungsreform}

\section{Manfred Röber}

Wandel der Verwaltung zwischen Erneuerungselan und Reformmüdigkeit

Jobst Fiedler

Erfahrungen, Bewertungen und Perspektiven der Verwaltungsreform aus der Beraterperspektive

Jörg Bogumil

Die Umgestaltung des Verhältnisses von Politik und Verwaltung

Hellmut Wollmann

Evaluierung von Verwaltungsmodernisierung

\section{Eckhard Schröter}

Europäischer Verwaltungsraum und Reform des öffentlichen Sektors

Register 


\section{Vorwort}

Mit der 3. Auflage des Handbuchs zur Verwaltungsreform aktualisieren wir ein Kompendium, das sich an Wissenschaft und Praxis gleichermaßen wendet. Die grundlegende Überarbeitung war durch den Fortgang der Verwaltungsreform seit 1998, als die 1. Auflage erschien, notwendig geworden. Wir haben dies aber auch zum Anlass genommen, einige Kapitel zu erweitern sowie ein neues, resümierendes Kapitel in das Buch einzufügen. Darüber hinaus ordnet eine neu hinzugenommene Einleitung den Prozess der Verwaltungsreform wissenschaftlich-konzeptionell ein und verknüpft dies mit der Struktur des Handbuchs.

In 56 Beiträgen werden Herkunft und Abgrenzung zentraler Begriffe und Konzepte der Verwaltungsreform geklärt, praktische Anwendungsfelder und Erfahrungen vermittelt sowie Entwicklungsperspektiven aufgezeigt. Auch soll von den einzelnen Begriffen her über ein Netz von Querverweisen sowohl ein Einstieg als auch ein Überblick über den Gesamtprozess ermöglicht werden. Im Vordergrund steht weniger der schnelle Zugriff, sondern mehr die systematische Erschließung des gesammelten „strategischen“ Wissens über die Verwaltungsreform. Zugleich sind die Beiträge im Durchschnitt auf einen Umfang reduziert, der es ermöglicht, sich in angemessener Zeit mit dem jeweiligen Thema zu beschäftigen. Der Aufbau des Handbuchs folgt der inneren Logik des Reformprozesses. Deshalb sind die Beiträge nicht alphabetisch geordnet, sondern nach sachlichen Kriterien in Kapiteln gruppiert. Der Leser wird in den Kapiteln von allgemeinen Darstellungen zu spezifischen Aspekten des Reformprozesses geführt.

Wir danken den Autorinnen und Autoren für ihre engagierte und verständnisvolle Mitarbeit, denn bei der Konzeption der 3. Auflage war wiederum viel Geduld erforderlich, unsere Interventionen und Kürzungen zu ertragen. Die einzelnen Beiträge enthalten nach unserer Einschätzung nunmehr eine Mischung aus dezidierter Spezialität und gerade so viel didaktischen Überschneidungen und Wiederholungen, dass ein kontinuierliches Weiterlesen ohne Zwang zu einem bestimmten Einstieg möglich sein sollte. Ein Register soll dabei helfen.

Der besondere Dank gilt den Mitarbeiterinnen und Mitarbeitern der Abteilung Sozialpolitik und Public Policy der Universität Hannover, Dr. Stefan Plaß, Marc Beer, M.A., Meral Adenli und Jennifer Faust, die sich bei der Redaktion des Handbuchs Verdienste erworben haben. Besonders erfreulich war, dass diese Arbeiten wie auch 
bei der 2. Auflage von der Hans-Böckler-Stiftung unterstützt wurden. Wir danken Frau Dr. Erika Mezger sehr herzlich dafür.

Hannover

Gelsenkirchen

Bremen

Berlin, im Juli 2004
Bernhard Blanke Stephan von Bandemer Frank Nullmeier Göttrik Wewer 


\section{Verwaltungsreform als Aufgabe des Regierens - Einleitung}

\section{Wissen für die ,lernende Verwaltung“ - Absichten und Adressaten}

In den letzten 10-15 Jahren haben Reformen der Verwaltung nicht nur an Umfang und Reichweite mit unterschiedlichen Geschwindigkeiten zugenommen, sie variieren auch zwischen den Ebenen des Staatsaufbaus (Bund, Länder, Kommunen) und zwischen Institutionen und Akteuren auf der gleichen Stufe. Die Bemühungen, die Verwaltung für ein neues Zeitalter vorzubereiten und nachhaltig reformfähig zu machen, setzten an sehr unterschiedlichen Teilbereichen an. Der Idee nach sollte diese Reform sich von früheren markant unterscheiden. Angelehnt an die Formeln des Business Management stand im Hintergrund vieler Konzepte und Instrumente die Vision eines ganzheitlichen und radikalen Schnitts und Umbaus. Tatsächlich zeigen die vorliegenden, noch stark anekdotischen Berichte über den Reformverlauf in Deutschland ein eher ernüchterndes Bild. Es wird von Ermüdung berichtet; aber die Erfolgsgeschichten sind auch nicht wenige. Einig sind sich die Beobachter weder über die Realitätseinschätzung noch in der Begriffs- und Konzeptionsbildung, selbst wenn sich mittlerweile wenigstens auf der instrumentellen Ebene so etwas wie ein Standardbaukasten herauskristallisiert.

Hierin liegt aber eine Gefahr: wenn sich die in den Reformprozess involvierten Praktiker, Berater und Wissenschaftler auf den kleinsten gemeinsamen Nenner verständlicher Modelle und standardisierter Elemente einigen, mag dies zu einer Beschleunigung der „Umsetzung“ von Reformen führen. Es kann sich aber auch um einen Prozess der „Sinnentleerung“ handeln, in welchem die Leitbilder und großen Ziele verloren gegangen sind und Herumwursteln in kleinteiligen Bereichen zum Alltag wird. Zwar wirken Innovationen nur dann nachhaltig, wenn sie in Routinen überführt werden. Nicht ausgereifte und missverstandene Innovationen führen als Routine jedoch zur Speicherung falscher Verhaltensweisen im Gedächtnis von Organisationen und Personen. Deshalb erscheint es allen kritischen Beobachtern dringend erforderlich, die im Laufe des Reformprozesses entstandenen Informationen zu sammeln, zu handlungsleitendem Wissen zu bündeln, dieses Wissen zu validieren und zu verbreiten, damit tatsächlich eine Reform- und Lernspirale in Gang gesetzt wird. Je mehr die an der Reform beteiligten Akteure unverbunden nebeneinander her lernen, umso mehr setzen sich Eigendynamiken durch, die den Verlust an strategischen Zielen und reformpolitischer Kontrolle sowie eine Delegitimation des ganzen Vorhabens nach sich ziehen können. Es kommt dann nicht zu jenen ,guten Ergebnissen“, welche im Sinne des Benchmarkings , das Salz in der Suppe“ jedes Veränderungsprozesses sein sollen. Das 
Handbuch wendet sich somit an alle, die weiterhin an kollektiven Lernprozessen für die Reform sowohl wissenschaftlich als auch im direkten Beratungsprozess sowie vor allem in der institutionalisierten Organisationspraxis beteiligt sind.

\section{Dimensionen und Facetten der Verwaltungsreform}

Wenn von einer „Sinnentleerung“ gesprochen wird, liegt die Frage nahe, welche Ergebnisse von der Verwaltungsreform erwartet wurden und werden. Hier scheiden sich die Geister, welche ein Reformprozess gerufen hat, der durch gesellschaftliche Makroprozesse, politische Programme und verwaltungsinterne Akteure sowie die Bürgerinnen und Bürger als Adressaten von Verwaltungshandeln getrieben wurde. International schälen sich durch die jeweilige nationale Tradition geprägte unterschiedliche Ursachen, verwaltungspolitische Reaktionen und Konzepte zur Reform heraus.

a) Verschiedene Begrifflichkeiten scheinen zunächst zu konkurrieren: Public Sector Reforms (Modernisierung des öffentlichen Sektors), „Staatsmodernisierung“ oder Public Management Reform sind Begriffe, die eine große Schnittmenge sowohl vom Gegenstand als auch von der Theorie her aufweisen, dennoch spezifische Differenzen markieren. Wir verwenden hier den Begriff der Verwaltungsreform, weil unser Buch nicht alle Dimensionen von Reformen des öffentlichen Sektors umfasst, wie z.B. Privatisierungen, Umbau des Sozialstaates, Steuerpolitik etc. Andererseits geht es hier nicht nur um die öffentliche Verwaltung im engeren Sinne, sondern die Reformkonzepte und -instrumente sind auf alle Verwaltungen anwendbar, die öffentliche Aufgaben erfüllen, seien sie halbstaatlich (wie in den Systemen der sozialen Sicherungen), freigemeinnützig oder privat. Die in unserem Handbuch systematisch bearbeiteten Elemente eines „Reformprojektes“ betten wir jedoch in die allgemeinere Diskussion um die Veränderung von „Staatlichkeit“ ein, denn hieraus entstammen Leitideen und Leitbilder, welche für Konzepte und Instrumente Sinn stiften.

b) Verwaltungsreform ist in allen, auf dem Weg zur Dienstleistungsgesellschaft befindlichen, industrialisierten Ländern ein Vorhaben, welches den langen Abschied von der inneren „Staatssouveränität“ möglicherweise beendet. Die moderne Verwaltung, wie sie im Idealtyp der Bürokratie im „Anstaltsstaat“ von Max Weber entfaltet wurde, hat seit der Fundamentaldemokratisierung zu Beginn des letzten Jahrhunderts, seit der Entstehung und massiven Ausweitung des Interventions- und Wohlfahrtsstaates, permanente Wandlungen vollziehen müssen. Selbst wenn in ihrem Kern die Behördenstruktur verblieben ist, fest verankert in der Verwaltungskultur mit ihren sozialisierenden Grundannahmen, Wertorientierungen des Personals und formellen Regeln und Routinen, ist diese ,anstaltsstaatliche“"Verwaltung am Kopf und an den Füßen entmachtet worden, und ihre Umweltbeziehungen haben sich aufgeweicht und sind zerfranst. Die demokratische Politik setzt oftmals vielfältige und miteinander im Konflikt liegende Ziele, die Bürgerinnen und Bürger sind keine gehorsamen Untertanen, und die Verwaltung muss neue Akzeptanz suchen. Beim Vollzug von Gesetzen und bei der Umsetzung von politischen Programmen ist die Verwaltung zum Verhandlungspartner 
von nichtstaatlichen Organisationen und Interessengruppen in jeweils unterschiedlichen Politikfeldern geworden.

c) Verwaltung und Verwaltungsreform stehen also in einem gänzlich anderen gesellschaftlichen Kontext als zu der Zeit, als die markanten bürokratischen Profile entwickelt und stabilisiert wurden, an denen sich heute noch das externe Umfeld, d.h. politische Führung, Bürgerinnen und Bürger und die Zivilgesellschaft insgesamt reiben. Das Profil der „Bürokratie als trivialer Maschine“, in der es immer nur einen Weg von oben nach unten oder umgekehrt gibt, entspricht schon lange nicht mehr der Realität und ist auch theoretisch seit Mitte des 20. Jahrhunderts obsolet. Nahezu sämtliche Themen der heutigen Verwaltungsreform: territoriale, funktionale, Organisations-, Personalund/oder Finanzreformen sind Dauerthemen in der Verwaltungsgeschichte und ,klassische' Schritte zu einer Verbesserung von Aufbau, Ablauf, Qualifikation, Mittelverwendung, Transparenz usw. Ob das gegenwärtige Reformprojekt nicht nur mit einer stets notwendigen Selbstreinigung der Bürokratie beschäftigt ist, sondern als Modernisierung verstanden werden kann, die ein neues post-bürokratisches Paradigma herausbildet, entscheidet sich an Themen aus der Debatte um eine Veränderung des Verhältnisses von Gesellschaft und Staat.

d) Die Makroinstitution Staat (dies gilt auch für den generischen Begriff von Government, wenn nicht einfach „Regierung“ gemeint ist) wurde in den vergangenen Jahrzehnten mit immer neuen differenzierenden Klassifikationen versehen, die meist nur eine Facette der Veränderung analytisch aufgegriffen haben. In der deutschen Reformpraxis tauchte als Leitbild zunächst der ,schlanke Staat“ auf, der später vom „Gewährleistungsstaat“ und vom ,aktivierenden Staat“ gefolgt wurde. Die Bundesregierung hat alle diese Klassifikationen 1999 unter dem Leitbild des „modernen Staates“ subsumiert. Diese Begriffe reflektieren jedoch eine zentrale Auseinandersetzung innerhalb der Reformgeschichte und sind in einem Oberbegriff nicht einfach zu ,versöhnen'.

Seit Mitte der 1970er-Jahre kämpfen verschiedene wissenschaftliche Strömungen und (partei-)politische Richtungen um eine Neudefinition von Umfang, Reichweite und Leistungstiefe von öffentlichen Aufgaben. Kaprizierte sich der „,schlanke Staat“ auf eine Reduktion von öffentlichen Aufgaben, thematisiert der „Gewährleistungsstaat“ eine andere Art der Aufgabenerfüllung und eine neue Aufgaben(ver)teilung zwischen Staat, Wirtschaft und Gesellschaft, so stellt der ,aktivierende Staat“ eine Teilung gemeinsam anerkannter und wahrgenommener öffentlicher Aufgaben zwischen kooperierenden Akteuren ins Zentrum seines Interesses. Solche differierenden Leitbilder führen zu unterschiedlichen Vorstellungen von Institutionen- und Organisationswandel.

e) Häufig sticht ein eher evolutionäres Konzept von Wandel hervor. Organisationstheorien sprechen von „lernenden Organisationen“ und „reflexiven Systemen“. Der Wandel ist im Sinne einer Anpassung an veränderte Umweltverhältnisse auch und gerade der Bürokratie immanent; allein schon aus Gründen der Herrschaftssicherung haben alle - auch die demokratischen - Landesherren Anpassungsreformen in Permanenz vollzogen. Intelligente Anpassung ist eine Form des Lernens. Eine andere, besonders in Zeiten massiver gesellschaftlicher und politischer Herausforderungen und Umbrüche geforderte Form, ist die einen Unterschied machende und nachhaltige Veränderung 
von Institutionen und deren Regelsystemen, meistens als erzwungene und das Überleben in einer nachhaltig neuen Umwelt sichernde Reform. Als höchste Stufe des Lernens wird die Fähigkeit von Organisationen angesehen, auch die Regeln der Umwelt $\mathrm{zu}$ beeinflussen und somit Regeln intern und extern selbst zu setzen. Ob die öffentlichen und andere an der öffentlichen Leistungserbringung beteiligten Verwaltungen dies können (sollen) ist zu problematisieren und bestenfalls als Analogie zu verstehen: denn die öffentliche Verwaltung sollte ja wohl nicht in der Lage sein, ihre Gesetzesbindung aufzuheben, freihändig neue Geschäftsfelder zu suchen oder sich selbst zu privatisieren. Dies mag auf den ersten Blick ein naiver Einwand sein, gleichwohl gehen viele Konzepte aus ökonomischer Sicht implizit von solchen Grundaxiomen aus. Für Verwaltungswissenschaftler, vor allem mit einem politikwissenschaftlichen Hintergrund, sind dies Kernaufgaben der demokratischen Politik und der in ihr gepflegten Verwaltungspolitik als eigenständigem Programmbereich. Aufgabenkritik ist letztlich eine Sache des Wahlvolkes, soweit es nicht um interne Geschäftsprozesse geht.

f) Verwaltungsreform als politisch geführter Prozess zielt auf eine Veränderung der Verwaltungskultur nach innen und in den Interaktionen mit der Umwelt. Die tradierte hierarchisch gesteuerte kontinuierliche Anpassung muss durch öffnende und offene Prozesse ergänzt werden, in welchen andere Typen der Koordination individueller und kollektiver Akteure angewandt werden wie Verhandlungen, Netzwerkbildung oder Wettbewerb. Diese Kombination verschiedener Koordinationsprozesse (oder Regelungsstrukturen) wird in der internationalen Literatur mit dem Begriff governance belegt. Als analytischer Begriff bezeichnet er die verschiedenen Möglichkeiten der Kombination von Koordinationstypen, deren normatives Ziel allerdings „,besseres Regieren“ (good governance) ist. Für die Verwaltungsreform ist dieses Konzept insoweit interessant und nützlich, als es einerseits Reformen in verschiedenen nationalen Kontexten vergleichbar macht, andererseits die Perspektive eröffnet, den Reformprozess selbst zu koordinieren und strategisch zu steuern.

\section{Spannungslinien im Reformprozess}

Die Divergenzen der Konzepte neuer Staatlichkeit treten an fundamentalen Spannungslinien der Reformpraxis in vergleichbaren Ländern auf und führen keineswegs zu modellartig auf längere Frist festzuschreibende Typen, wie sie immer wieder ins Zentrum der Literatur gestellt werden (wie angelsächsischer, kontinentaleuropäischer oder skandinavischer Typ). Häufig genug haben einzelne Länder im Zeitverlauf in ihrem langfristig pfadabhängigen Handlungskorridor die Wege der Public Sector Reform gewechselt. Konkrete Erfolge oder Misserfolge von Projekten führten zu Revisionen und teilweise ,dramatischen" Neuerungen. Auch Regierungswechsel machten einen Unterschied.

a) Wenn in der englischsprachigen Literatur lange Zeit die Öffnung des Public Sectors für den Wettbewerb propagiert und in einzelnen Ländern praktiziert wurde, bis hin zu radikalen Privatisierungen, so wurde dies häufig genug als das eigentliche, generische 
Reformkonzept für alle industrialisierten Länder verstanden und gefordert. Wenn sich ein Land diesem Trend - aus welchen Gründen auch immer - widersetzte, galt es als , altmodisch“. Die Zeiten ändern sich: viele „Vermarktlichungen“ sind z.B. in Großbritannien zurückgenommen und durch andere Konzepte ersetzt worden, die auf einen anderen Koordinationstyp zurückgreifen, nämlich Netzwerke. Heute stehen partnership und joining up-Ideen im Vordergrund.

b) Hierarchieabbau, Dezentralisierung und Autonomisierung von ehemaligen Behörden zu Agenturen (agencies) gelten als ein anderes generisches Konzept für Public Sector Reforms, und viele Länder haben mit unterschiedlichen Ansätzen konkrete Reformen entlang dieser Devise durchgeführt. Dennoch kann man kaum eine dieser Reformen zum überall anwendbaren Modell (auch nicht das schwedische) hochstilisieren. Dezentralisierung und Autonomisierung sind von hochkomplizierten Vertragswerken begleitet worden, die ihrerseits einen zunehmenden Zentralisierungsschub von Auditierungen und Controlling nach sich ziehen. Das Dilemma scheint unausweichlich, solange die Ergebnisbewertung bei der Erfüllung öffentlicher Aufgaben nicht einfach einem anonymen Markt und einzelnen Kundensegmenten überlassen werden kann, sondern im demokratischen Staat die Wahlbürger das letzte Wort haben.

c) „Marketization“ oder kooperative Vernetzung einerseits, Dezentralisierung oder Rezentralisierung andererseits erscheinen als zwei grundlegende Spannungslinien. Eine andere, ebenso wichtige Spannung entsteht zwischen Öffnung und Schließung des „,bürokratischen" Machtbereiches. Öffnung ist erwünscht (nicht nur durch Wettbewerb) vor allem im Interesse des Organisationslernens; wie weit sich die öffentliche Verwaltung öffnen darf, ist wiederum umstritten. Das Risiko der einseitigen Indienstnahme für spezifische Interessen muss gegen den Vorteil der höheren Interessenberücksichtigung der Bürgerinnen und Bürger allgemein und der gesteigerten Problemlösungskompetenz ,vor Ort' abgewogen werden. Insbesondere für das Personal ergeben sich vollkommen neue Herausforderungen und oft mehrdeutige Orientierungen. Hier lässt sich am deutlichsten greifen, ob eine grundlegende „Modernisierung“ der Verwaltung angestrebt oder erreicht wurde und welche (prekären) Konsequenzen dieser Prozess mit sich gebracht hat.

d) Eine Voraussetzung ist zweifelsohne, dass Organisations- und individuelles Lernen auf diesem Weg nicht gestört oder gar unterdrückt werden durch die notorischen $\mathrm{Fi}$ nanzierungsprobleme im öffentlichen Sektor. Viele Autoren gehen mittlerweile davon aus, dass diese Probleme die angestrebte grundlegende Erneuerung der Verwaltung konterkariert haben. Reformen des öffentlichen Haushalts- und Rechnungswesens hatten nach allgemeiner Ansicht in der deutschen Verwaltungsreform des vergangenen Jahrzehnts ein Übergewicht. Von einem ganzheitlichen, wenngleich nicht zeitgleich überall geschlossen durchgeführten Reformkonzept her gesehen, sollten die auf Sparsamkeit und Effizienz abstellenden Instrumente in einem logischen Kontext mit anderen Instrumenten stehen. Sie sollen weder rein der Haushaltssanierung dienen, noch zum bürokratischen Selbstzweck werden. Ihre zielführende Kategorie sollte die Effektivität im Sinne der Zielerreichung öffentlicher Programme sein. Und diese wiederum bemisst sich nach den Ergebnissen und Wirkungen des Verwaltungshandelns. Abgekoppelt von einer solchen Orientierung, die aber selbst wiederum innerhalb der Orga- 
nisation und beim Personal die Neuorientierung voraussetzt, richten neue Instrumente möglicherweise mehr Schaden an als sie Nutzen stiften.

\section{Konzepte und Instrumente im pluridisziplinären Diskurs}

Eine Ursache für die Isolierung und teilweise Dominanz der betriebswirtschaftlichen Konzepte in der Verwaltungsreform und vor allem ihres Missverständnisses bei den Anwendern in Deutschland (von anderen Ländern wird Ähnliches berichtet) könnte sein, dass hier zu Lande die Konzepte zur Modernisierung des öffentlichen Sektors zu einem Zeitpunkt ,importiert' wurden, als Neuseeland, Großbritannien und mitunter die USA als Vorbilder galten. Deren Botschaft wurde zunächst vom Managerialismus der 1980er-Jahre geprägt (Reagan, Thatcher), der recht krude dem „bureau“ und den Bürokraten den Kampf angesagt hatte. Leitidee war die komplette Übertragung von privaten Managementtechniken auf den Public Sector, in der Annahme, zu managen wäre überall das Gleiche. Oder anders formuliert: es gäbe keine spezifische Differenz des öffentlichen Sektors, mit der Ausnahme, dass die Ziele politisch und nicht marktlich definiert würden. Die Idee vom Staat als Dienstleistungsunternehmen hatte auch die Reformen unter Clinton und Gore in den USA bestimmt und mit der Analogie eines businesslike Public Management weitere Fehlschlüsse ermöglicht. Businesslike but not like business lautete die Reaktion in Großbritannien in den 1990er-Jahren, mit der die spezifische Differenz des öffentlichen Sektors herausgestellt werden sollte, ohne die Impulse aus dem Managerialismus zu negieren.

Aus dem Spannungsfeld zwischen krudem Managerialismus, wissenschaftlichen Reformtheorien und reflektierter Erfahrung von Verwaltungspolitikern und den einzelnen Verwaltungsabteilungen entstanden hybride Konzepte, die einen modellartigen Transfer ermöglichen. Dadurch wurde einerseits deren Komplexität gesteigert (und ein neuer, mitunter verwirrender Markt an Literatur generiert). Andererseits erzwang ein international intensivierter Diskurs (vor allem über die OECD, die eine Art „offener Koordinierung“ praktizierte) eine stetige Systematisierung. Ausgehend vom Begriff New Public Management scheint sich eine paradigmatische Strömung herausgebildet zu haben, die sich als Teil einer neuen Regierungslehre versteht. Sie zeichnet sich dadurch aus, dass die Grenzziehungen zwischen den unterschiedlichen wissenschaftlichen Fachdisziplinen aufgeweicht und Erkenntnisse und Erfahrungen integriert werden. Unterschiedliche wissenschaftliche Rationalitäten werden in einem durch die intensive Beteiligung an Design und Evaluation von Reformen bestimmten Lernprozess verknüpft, der selbst eine erkennbare Eigendynamik entwickelt hat.

In Deutschland ist der Diskurs noch deutlich fachwissenschaftlich konturiert. Die im Staats- und Verwaltungshandeln verschmolzenen unterschiedlichen Rationalitäten (legale Richtigkeit und Gerechtigkeit, Wirtschaftlichkeit, Wirksamkeit und demokratische Verantwortung u.a.) werden häufig genug isoliert abgebildet. Dadurch besteht aber die Gefahr, dass sich aus dem Theorieangebot des New Public Management, welches an sich keine Präferenz für bestimmte nationale Reformpfade logisch nahe legt, doch der eher ökonomisierende Trend in den Vordergrund schiebt, mit seiner Beto- 
nung von Flexibilität, Effizienz und (interner) Effektivität - mit dem Bürger als Kunden im Schlepptau. Die deutsche Verwaltungstradition scheint sich demgegenüber nur als Beharrung durchsetzen zu können. Dies ist bedauerlich, weil es durchaus sein könnte, dass die alten Werte einer auf Rechtsstaatlichkeit und Gleichbehandlung, verlässliche und transparente Verfahren sowie (gerichtlich) überprüfbare Ergebnisse abgestellten Verwaltungskultur einer Idee „,neuer“ Staatlichkeit keineswegs im Wege stehen. Denn der wesentliche Reformtreiber ist die um sich greifende Einsicht, dass öffentliche Verwaltungen Teil des Regierens sind und spezifische Leistungen verantwortlich für alle Bürgerinnen und Bürger erbringen müssen. Die verschiedenen Funktionszuschreibungen der Verwaltung wie Gesetzesvollzug, Politikumsetzung (policy implementation) oder öffentliche Leistungserbringung (public services delivery) sind nicht gegeneinander abzusetzen, sondern Dimensionen eines Prozesses. Je schärfer dieser Gedanke in den Vordergrund gerückt werden kann, desto mehr müssten auch die wissenschaftlichen Disziplinen kooperieren, denn nur so entsteht ein mehrschichtig rationales post-bürokratisches Modell.

Wir haben in diesem Handbuch viele Autorinnen und Autoren aus vielen Richtungen und Praxisfeldern zu Wort kommen lassen, um die Vielfalt und Breite des verwaltungspolitischen Diskurses sichtbar zu machen. Gleichzeitig steht diese systematisch gegliederte Artikelsammlung jedoch nicht in einem einheitlichen Rahmen, aus welchem sich deduktiv Reformschritte in einer logischen oder zeitlichen Abfolge unmittelbar ableiten ließen. Die handlungsorientierte Integration in den jeweiligen Kontext soll dem Nutzer überlassen werden.

\section{Literatur}

Bovaird, Tony/Löffler, Elke (eds.), 2003: Public Management and Governance, London.

Christensen, Tom/Laegreid, Per (eds.), 2002: New Public Management. The transformation of ideas and practice, Aldershot.

Hesse, Joachim Jens/Benz, Arthur, 1990: Modernisierung des Staates, Baden-Baden.

Jann, Werner u.a., 2004: Status-Report Verwaltungsreform. Eine Zwischenbilanz nach zehn Jahren (Modernisierung des öffentlichen Sektors, Bd. 24), Berlin.

Klages, Helmut, 2003: Nachhaltige Verwaltungsmodernisierung. Eine Bilanz nach zwölf Jahren Neuer Steuerung, in: Verwaltung und Management, 9. Jg., Heft 1, S. 4-12.

Klimecki, Rüdiger/Müller, Werner (Hrsg.), 1999: Verwaltung im Aufbruch. Modernisierung als Lernprozess, Zürich.

König, Klaus (Hrsg.), 2002: Deutsche Verwaltung an der Wende zum 21. Jahrhundert, Baden-Baden.

Mc Laughlin, Kate/Osborne, Stephen/Ferlie, Ewan (eds.), 2002: New Public Management. Current trends and future prospects, London.

Naschold, Frieder/Bogumil, Jörg, 2000: Modernisierung des Staates. New Public Management in deutscher und internationaler Perspektive, 2. vollständig aktualisierte u. stark erw. Aufl., Opladen.

OECD (Hrsg.), 2000: Government of the Future, Paris.

Peters, B. Guy/Pierre, Jon (eds.), 2002: Handbook of Public Administration, London.

Pollitt, Christopher/Bouckaert, Geert, 2004: Public Management Reform. A Comparative Analysis, 2. ed., Oxford. 